\title{
Concordância interobservadores dos métodos estáticos de avaliação dos tipos de pisada em praticantes de corrida*
}

\section{Interobserver Agreement of the Static Methods of Evaluating the Types of Footprint in Runners}

\author{
Rafael Rocha Macedo ${ }^{1,2}$ Thiago Kazunori Neves Kanashiro ${ }^{10}$ \\ Gustavo Henrique Ramalho de Mattos ${ }^{10}$ Alexandre Alcaide ${ }^{3(0)}$ Luciano Miller Reis Rodrigues ${ }^{2(0)}$ \\ Eiffel Tsuyoshi Dobashi ${ }^{10}$ \\ ${ }^{1}$ Hospital IFOR - Rede D’Or São Luiz, São Bernardo do Campo, SP, \\ Endereço para correpondência Rafael da Rocha Macedo, MD, Rua \\ Brasil \\ ${ }^{2}$ Disciplina de Ortopedia e Traumatologia, Faculdade de Medicina do \\ Piranhas, 50, apto 104, Torre 1, Santo André, SP, 09050-080, Brasil \\ ABC, Santo André, SP, Brasil \\ ${ }^{3}$ Clínica de Ortopedia e Recuperação Desportiva, Santo André, SP, \\ (e-mail: rrochamacedo@yahoo.com.br). \\ Brasil
}

Rev Bras Ortop 2020;55(4):490-496.

\section{Resumo \\ Palavras-chave \\ - pé \\ - corrida moderada \\ - exame físico \\ Objetivo Avaliar a acurácia dos métodos de diagnóstico interobservadores do tipo de pisada em atletas corredores utilizando três métodos de avaliação: exame físico, podoscopia e baropodometria comparando com a medida radiográfica dos ângulos de Meary e pitch do calcâneo. \\ Métodos Trata-se de estudo transversal de atletas que praticam corrida. Os critérios de inclusão foram: indivídulos com idade mínima de 18 anos e máxima de 65 anos de ambos os sexos; indivídulos hígidos, sem comorbidades que interfiram no desempe- nho da corrida; praticantes regulares que realizam corrida ao menos duas vezes na semana; índice de massa corpórea entre 18,5 e $29,99 \mathrm{Kg} / \mathrm{m}^{2}$; aceitação dos termos de consentimento livre e esclarecido (TCLE). Os critérios de não inclusão compreenderam: presença de lesões prévias ou ativas que comprometam a atividade esportiva; cirurgias prévias dos pés; obesidade. Foram selecionados 40 pacientes, sendo $29(72,5 \%)$ do sexo masculino e 11 (27,5\%) do sexo feminino cuja média das idades foi de 39 anos (mínimo de 19 anos e máximo de 61 anos). $O$ índice de massa corpórea (IMC) dos 40 pacientes variou de 21,00 a $29,99 \mathrm{Kg} / \mathrm{m}^{2}$ (média de $25,48 \mathrm{Kg} / \mathrm{m}^{2}$ com desvio-padrão de 2,39 $\mathrm{Kg} / \mathrm{m}^{2}$ e mediana de $25,50 \mathrm{Kg} / \mathrm{m}^{2}$ ). Excluímos os que apresentaram valor superior a $29,99 \mathrm{Kg} / \mathrm{m}^{2}$. A frequência de corrida variou de 2 a 5 vezes por semana (média de 3,13 vezes por semana com desvio-padrão de 0,79 vezes por semana e mediana de 3 vezes por semana). Foram realizados exame físico, podoscopia e baropodometria, e sua}

\footnotetext{
Trabalho realizado na Clínica de Ortopedia e Recuperação Desportiva, Santo André, SP, Brasil.
}

recebido

06 de Fevereiro de 2019

aceito

13 de Setembro de 2019
DOI https://doi.org/

$10.1055 / \mathrm{s}-0039-3402464$. ISSN $0102-3616$.
Copyright $\odot 2020$ by Sociedade Brasileira License terms de Ortopedia e Traumatologia. Published by Thieme Revinter Publicações Ltda, Rio de Janeiro, Brazil 
avaliação foi feita por quatro examinadores; além disso, os resultados foram comparados com a classificação radiográfica do tipo de pisada obtida através da mensuração dos ângulos de Meary e pitch do calcâneo.

Resultados A concordância interobservadores destes parâmetros foi verificada pelo índice de concordância de Kappa ponderado, segundo o qual obtivemos uma concordância significante entre os participantes, levando-se em consideração o exame físico, podoscopia e baropodometria e de acordo com o índice Kappa. A concordância foi marginal quando comparados os resultados dos três métodos com a classificação angular radiográfica.

Conclusão Obtivemos uma concordância excelente entre os observadores ao considerarmos o exame físico, a podoscopia e a baropodometria para o diagnóstico do tipo pisada em corredores. Porém, quando comparados os resultados obtidos com as medidas radiográficas, a concordância para o diagnóstico do tipo da pisada foi baixa.

\section{Abstract}

Keywords

- foot

- moderate running

- physical examination
Objective To evaluate the accuracy of interobserver diagnostic methods of the type of footprint among running athletes using three evaluation methods: physical examination, podoscopy, and baropodometry compared with radiographic measurement of Meary angles and calcaneal pitch.

Methods This is a cross-sectional study of athletes who practice running. The inclusion criteria were: individuals with minimum age of 18 years and maximum age of 65 , male or female, healthy and without comorbidities that interfere with running performance; regular practitioners who run at least twice a week; body mass index between 18.5 and $29.99 \mathrm{Kg} / \mathrm{m}^{2}$; acceptance of the written informed consent form (WICF). The non-inclusion criteria included: presence of previous or active injuries that compromise sports activity; previous foot surgeries; obesity. Forty patients were selected, $29(72.5 \%)$ male and $11(27.5 \%)$ female, whose mean age was 39 years (minimum 19 years and maximum 61 years). The body mass index (BMI) of the 40 patients ranged from 21.00 to $29.99 \mathrm{~kg} / \mathrm{m}^{2}$ (mean $25.48 \mathrm{~kg} / \mathrm{m}^{2}$ with standard deviation of $2.39 \mathrm{~kg} / \mathrm{m}^{2}$ and a median of $25.50 \mathrm{~kg} / \mathrm{m}^{2}$ ). We excluded those with values above 29.99. Running frequency ranged from 2 to 5 times per week (average 3.13 times per week, with standard deviation of 0.79 times per week and median of 3 times per week). Physical examination, podoscopy, and baropodometry were performed, and their evaluation was made by 4 examiners. Additionally, the results were compared with the radiographic classification of the footprint type obtained by measuring the Meary angles and the calcaneal pitch.

Results The interobserver agreement of these parameters was verified by the weighted Kappa agreement index, in which we obtained a significant agreement between the participants considering physical examination, podoscopy, and baropodometry, and according to the Kappa index. The agreement was marginal when comparing the results of the three methods with the radiographic angular classification.

Conclusion We obtained excellent agreement among observers when considering physical examination, podoscopy, and baropodometry for the diagnosis of the footprint type among runners. However, when comparing the results obtained with the radiographic measurements, the agreement for the diagnosis of footprint type was low.

\section{Introdução}

A corrida, como atividade física, tem ganhado grande destaque ao longo dos últimos anos pela sua fácil acessibilidade e baixo custo. Esta modalidade também proporciona aos seus praticantes uma grande independência por se tratar de um esporte individual. Além disso, traz benefícios perceptíveis, como a melhora da qualidade cardiorrespiratória e do tônus muscular. ${ }^{1}$ 
Apesar dos benefícios destacados, encontramos uma taxa de ocorrência de $79 \%$ de lesões nos membros inferiores, especialmente nos pés e joelhos, em praticantes de corrida contínua. $^{2}$

Para a melhora do rendimento e da resistência corporal, a prática deste esporte requer fomento de informações científicas apropriadas para que a sua perfeita prática possa ser desenvolvida. $^{3}$

Considerando especificamente o pé, este segmento corporal de contato do corpo com o solo é provido de rica quantidade de receptores cutâneos, exteroceptores e de propriocepção. Este fato colabora com o equilíbrio e ajusta a postura corporal durante a posição ereta. ${ }^{4}$

São descritos três tipos de padrão para os pés, quanto à configuração do arco medial (pé plano, côncavo, e normal), com quatro tipos de pisada (neutra, supinada, pronada, e acentuadamente pronada) sendo que tais variações são individuais. ${ }^{5,6}$

A análise clínica da pisada em corredores deve ser realizada por meio de um exame físico detalhado dos pés auxiliado pela podoscopia e baropodometria.

A podoscopia é utilizada para estudar a área de contato dos pés com relação ao solo sob visão direta, ${ }^{7}$ enquanto que a barodopodometria eletrônica visa mensurar e comparar as pressões distribuídas em diferentes áreas da região plantar do pé na posição estática ou durante a marcha. ${ }^{8}$

Diversos autores têm se utilizado desses recursos para avaliar os problemas clínicos dos pés com o intuito de identificar anormalidades para proporcionar adequadamente mudanças na distribuição da pressão plantar. Também é visto como método seguro que auxilia o planejamento e a escolha terapêutica adequada para os pés. ${ }^{9}$

Outro recurso amplamente utilizado é a radiografia dos pés com carga, que tem por objetivo avaliar alterações do alinhamento e dos espaços articulares, sendo possível também promover a análise das características do arco medial do pé, bem como classificar o tipo da pisada através de mensurações angulares. ${ }^{10}$

Quando fizemos uma análise crítica da literatura considerando o binômio corrida e os diferentes métodos de diagnóstico complementar, encontramos um número reduzido de artigos. Portanto, o presente estudo foi realizado com o objetivo primário de verificar a concordância interobservadores considerando o exame físico ortopédico do pé, podoscopia e baropodometria e, secundariamente, fazer uma comparação dos resultados com a classificação radiográfica obtida através da mensuração dos ângulos de Meary e pitch do calcâneo nas radiografias em perfil com carga.

\section{Material e método}

O presente estudo foi submetido ao comitê de ética em pesquisa da Plataforma Brasil pelo CAAE 97161218.5.0000.5625 e aprovado para realização de acordo com o parecer 2.946.418.

Trata-se de estudo transversal que pretende avaliar a concordância interobservadores analisando as características da pisada estática de atletas que praticam corrida e comparando os resultados com os da classificação radiográfica.
Os critérios de inclusão para os participantes deste estudo foram:

1) Ter idade mínima de 18 anos e máxima de 65 anos de ambos os sexos.

2) Pacientes hígidos, sem comorbidades que interfiram no desempenho da corrida.

3) Praticantes regulares que realizam corrida ao menos duas vezes na semana.

4) Oíndice de massa corpórea (IMC) entre 18,5 e 29,99 Kg/m² (peso normal e sobrepeso)

5) Aceitar os termos e assinar o termo de consentimento livre e esclarecido (TCLE).

A proposição de participacão do paciente foi feita em entrevista pessoal, ocasião em que os pesquisadores explanaram a importância e o objetivo do estudo, certificando-se, posteriormente, da compreensão correta e total do sujeito, a fim de evitar vício quanto ao seu consentimento. Destaca-se que a recusa à aceitação e assinatura do TCLE ou retirada do consentimento ao mesmo poderia ocorrer a qualquer momento, sem o acarretamento de consequências de qualquer espécie ao indivíduo.

Os critérios de não inclusão compreenderam:

1) Portadores de lesões prévias ou ativas que comprometam a atividade esportiva, tais como neuropatias periféricas, degenerativas, sequelas de trauma, doenças inflamatórias locais ou sistêmicas, etc.

2) Cirurgias prévias dos pés.

3) Corredores com valor de IMC superior a $30,00 \mathrm{Kg} / \mathrm{m}^{2}$.

Foram então compilados 40 pacientes, sendo $29(72,5 \%)$ do sexo masculino e 11(27,5\%) do feminino, cuja média das idades foi de 39 anos (mínimo de 19 anos e máximo de 61 anos).

O IMC dos 40 pacientes variou de 21,00 a $29,99 \mathrm{Kg} / \mathrm{m}^{2}$ (média de $25,48 \mathrm{Kg} / \mathrm{m}^{2}$ com desvio-padrão de $2,39 \mathrm{Kg} / \mathrm{m}^{2}$ e mediana de $25,50 \mathrm{Kg} / \mathrm{m}^{2}$ ). Excluímos os que apresentaram valor superior a $30,00 \mathrm{Kg} / \mathrm{m}^{2}$.

A frequência de corrida variou de 2 a 5 vezes por semana (média de 3,13 vezes por semana com desvio-padrão de 0,79 vezes por semana e mediana de 3 vezes por semana).

Elaboramos um protocolo de atendimento em que foram contempladas algumas variáveis consideradas importantes pelos pesquisadores. Foram selecionados três parâmetros de avaliação, sendo eles o exame físico, a podoscopia e a baropodometria.

\section{Método de avaliação clínica:}

1.1 Inspeção estática do pé e tornozelo com o paciente em cima de uma plataforma plana de 1 metro de altura com o indivíduo em ortostase obedecendo marcações prévias no solo com intuito de padronizar a posição para o exame, os examinadores posicionados um de cada vez todos na mesma angulação para a análise para minimizar os erros em virtude da angulação assumida no ato da verificação. Os seguintes parâmetros forma analisados no exame físico:

- Posição do retropé - esta mensuração foi realizada na posição ortostática, pela vista posterior onde os eixos do retropé e tornozelo foram encontrados da seguinte maneira: para se traçar o eixo do retropé foi localizado 
o centro clínico do calcâneo e o centro do tornozelo para o traçado de uma reta. Do centro do tornozelo para amassa tricipital foi traçada uma segunda reta. 0 cruzamento das mesmas foi aferido com o uso de goniômetro para aferir a medida em graus desta variável. (neutro, cavo ou valgo)

- altura da abóbada plantar - utilizamos o método de inspeção não quantitativa, onde classificamos o pé como plano, neutro, ou cavo pela visualização direta da altura da abóbada plantar. É um método observacional onde visualizamos a porção interna do mediopé onde podem ser encontradas três situações: pé plano (todo mediopé apoiado na plataforma), pé neutro (região medial com apoio parcial do arco medial na plataforma), ou pé cavo (mediopé totalmente sem apoio na plataforma).

\section{Método de avaliação podoscópica:}

Todos os pacientes realizaram o exame de podoscopia e foram observados pelos quatro examinadores individualmente que diagnosticaram o apoio estático bipodal dos pés em três categorias: neutra, pronada, ou supinada.

Utilizamos a classificação de Valente, de acordo com a qual, para uma pisada neutra, a largura do istmo corresponderia a menos da metade da largura total do antepé. Caso a largura do istmo viesse a superar a metade da largura do antepé o diagnóstico seria de um pé plano. Caso a largura do istmo fosse inferior a 1/3 seria diagnosticado o pé cavo.

Todos os pacientes foram examinados em ortostase de frente e de costas, respeitando a mesma posição pré-estabelecida com marcas no aparelho para evitar posicionamentos errôneos ou involuntários.

\section{Método de avaliação baropodométrica:}

Os voluntários também foram submetidos a um exame de baropodometria estática, sendo utilizado um aparelho da marca FOOTWORK Arkipelago (Arkipelago, São Paulo, SP, Brasil), com 2.704 captadores com pressão máxima de 100 $\mathrm{N} / \mathrm{cm}^{2}$ por captador. Os sinais elétricos foram captados e enviados para um computador que gerou uma imagem que foi analisada pelos observadores, definindo, assim, o tipo de pisada para cada corredor.

\section{Método de avaliação radiográfica}

Os voluntários realizaram uma radiografia dos pés com carga na incidência perfil para mensuração dos ângulos de Meary e pitch do calcâneo feita previamente por um dos examinadores e conferida por um segundo e, de acordo com tais angulações, deram o diagnóstico para cada pisada, sendo: pronada com pitch menor que 10 graus e Meary menor que -4 graus; supinada com pitch maior que 30 graus e Meary maior que +4 graus; neutra (normal na literatura) com pitch de 10 a 30 graus e Meary $-4 a+4$ graus, de acordo com a classificação angular já consagrada na literatura. ${ }^{11}$ Os métodos para mensuração estão descritos na - Figura 1.

\section{Análise estatística}

Inicialmente todas as variáveis foram analisadas descritivamente. Para as variáveis quantitativas esta análise foi feita através da observação dos valores mínimos e máximos e do cálculo de médias, desvios-padrão e mediana. Para as variáveis qualitativas foram calculadas as frequências absolutas e relativas.

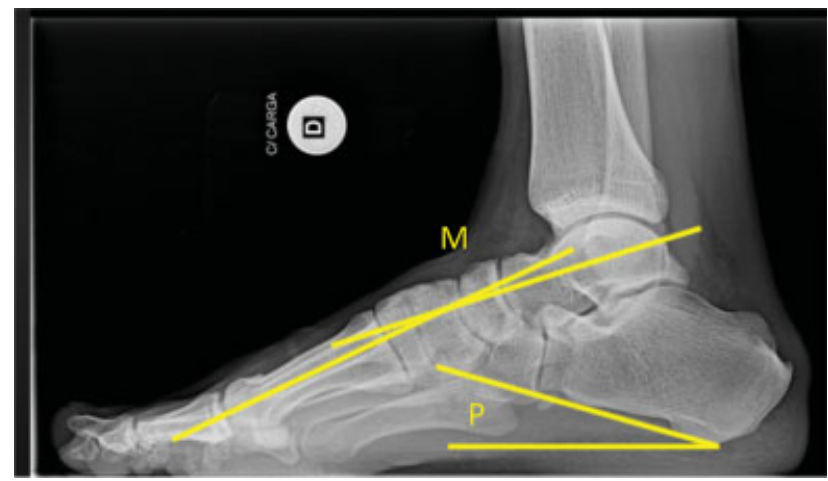

Fig. 1 Medidas dos ângulos Meary (M) e pitch do calcâneo (P).

A concordância entre os observadores foi avaliada por meio do índice de concordância Kappa ponderado (K), ${ }^{12}$ cuja interpretação é:

$\mathrm{K}>0,75$ concordância excelente

$0,4 \leq \mathrm{K} \leq 0,75$ concordância boa

$0 \leq \mathrm{K} \leq 0,4$ concordância marginal

\section{Resultados}

Foram avaliados 40 pacientes com idade entre 19 e 61 anos (média de 39 anos com desvio-padrão de 10,68 anos e mediana de 39,50 anos). Vinte e nove (72,5\%) pacientes eram do sexo masculino.

O IMC dos 40 pacientes variou de 21,00 a $29,99 \mathrm{Kg} / \mathrm{m}^{2}$ (média de $25,48 \mathrm{Kg} / \mathrm{m}^{2}$ com desvio-padrão de $2,39 \mathrm{Kg} / \mathrm{m}^{2}$ e mediana de $25,50 \mathrm{Kg} / \mathrm{m}^{2}$ ).

A frequência de corrida variou de 2 a 5 vezes por semana (média de 3,13 vezes por semana com desvio-padrão de 0,79 vezes por semana e mediana de 3 vezes por semana).

O pitch do calcâneo observado pela radiografia variou de $12^{\circ}$ a $34^{\circ}$ (média de $22,70^{\circ}$ com desvio-padrão de $5,54^{\circ}$ e mediana de $22,00^{\circ}$ ).

$\mathrm{Na}$ - Figura 2 apresentamos a distribuição de freqüências dos 40 pacientes, segundo a classificação radiológica.

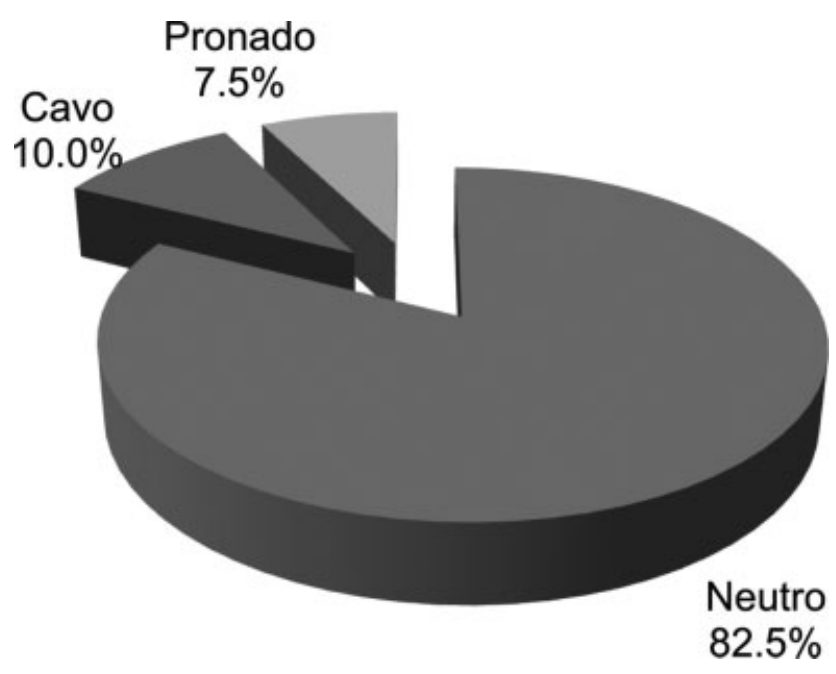

Fig. 2 Frequências relativas dos 40 pacientes segundo a classificação radiológica. 
Tabela 1 Frequências absolutas e relativas das avaliações do quatro observadores, segundo o exame físico, a podoscopia e a baropodometria

\begin{tabular}{|c|c|c|c|c|c|c|c|c|c|}
\hline & & \multicolumn{8}{|c|}{ Observador } \\
\hline & & \multicolumn{2}{|l|}{1} & \multicolumn{2}{|l|}{2} & \multicolumn{2}{|l|}{3} & \multicolumn{2}{|l|}{4} \\
\hline Exame & & $n$ & $\%$ & $\mathrm{n}$ & $\%$ & $n$ & $\%$ & $n$ & $\%$ \\
\hline \multirow[t]{3}{*}{ Físico } & Neutro & 19 & 47,5 & 17 & 42,5 & 18 & 45,0 & 22 & 55,0 \\
\hline & Cavo & 6 & 15,0 & 5 & 12,5 & 7 & 17,5 & 7 & 17,5 \\
\hline & Pronado & 15 & 37,5 & 18 & 45,0 & 15 & 37,5 & 11 & 27,5 \\
\hline \multirow[t]{3}{*}{ Podoscopia } & Neutro & 25 & 62,5 & 31 & 77,5 & 18 & 45,0 & 19 & 47,5 \\
\hline & Cavo & 8 & 20,0 & 5 & 12,5 & 12 & 30,0 & 7 & 17,5 \\
\hline & Pronado & 7 & 17,5 & 4 & 10,0 & 10 & 25,0 & 14 & 35,0 \\
\hline \multirow[t]{3}{*}{ Baropodometria } & Neutro & 17 & 42,5 & 22 & 55,0 & 18 & 45,0 & 18 & 45,0 \\
\hline & Cavo & 15 & 37,5 & 13 & 32,5 & 15 & 37,5 & 13 & 32,5 \\
\hline & Pronado & 8 & 20,0 & 5 & 12,5 & 7 & 17,5 & 9 & 22,5 \\
\hline
\end{tabular}

Foram realizadas avaliações destes 40 pacientes através do exame físico, da podoscopia e da baropodometria por quatro observadores. Na - Tabela 1 apresentamos estas avaliações.

$\mathrm{Na}$ - Tabela 2 apresentamos os valores do índice de concordância Kappa ponderado entre os quatro observadores no exame físico. Através destes valores podemos concluir que o observador 2 apresenta excelente concordância com os observadores 1 e 3 e entre os demais observadores temos boa concordância.

Na - Tabela 3 apresentamos os valores do índice de concordância Kappa ponderado entre os quatro observadores na Podoscopia. Através destes valores podemos concluir que houve uma boa concordância entre todos os observadores quando comparados entre si.

$\mathrm{Na}$ - Tabela 4 apresentamos os valores do índice de concordância Kappa ponderado entre os quatro observadores na baropodometria. Através destes valores podemos concluir que o observador 1 apresenta boa concodância com os observadores 2,3 e 4 . O observador 2 apresenta excelente concordância com o observador 3 e boa concordância com o observador 4 e o observador 3 apresenta excelente concordância com o observador 4 .

Tabela 2 Valor do índice Kappa ponderado e respectivo intervalo de confiança a $95 \%$ dos quatro observadores em relação ao exame físico

\begin{tabular}{|l|l|l|l|}
\hline & \multicolumn{3}{|l|}{ Observador } \\
\hline Observador & 2 & 3 & 4 \\
\hline 1 & 0,78 & 0,73 & 0,70 \\
\hline & $(0,65 ; 0,93)$ & $(0,56 ; 0,89)$ & $(0,53 ; 0,87)$ \\
\hline 2 & & 0,77 & 0,61 \\
\hline & & $(0,65 ; 0,90)$ & $(0,44 ; 0,78)$ \\
\hline 3 & & & 0,69 \\
\hline & & & $(0,52 ; 0,86)$ \\
\hline
\end{tabular}

Na - Tabela 5 apresentamos os valores do índice de concordância Kappa ponderado dos quatro observadores em relação à radiografia. Através destes valores podemos concluir que os observadores apresentam concordância marginal entre os exames realizados e a radiografia.

Tabela 3 Valor do índice Kappa ponderado e respectivo intervalo de confiança a 95\% dos quatro observadores em relação à podoscopia

\begin{tabular}{|l|l|l|l|}
\hline & \multicolumn{3}{|l|}{ Observador } \\
\hline Observador & 2 & 3 & 4 \\
\hline 1 & 0,50 & 0,65 & 0,56 \\
\hline & $(0,26 ; 0,74)$ & $(0,47 ; 0,83)$ & $(0,37 ; 0,75)$ \\
\hline 2 & & 0,45 & 0,41 \\
\hline & & $(0,23 ; 0,67)$ & $(0,20 ; 0,61)$ \\
\hline 3 & & & 0,70 \\
\hline & & & $(0,56 ; 0,85)$ \\
\hline
\end{tabular}

Tabela 4 Valor do índice Kappa ponderado e respectivointervalo de confiança a $95 \%$ dos quatro observadores em relação à baropodometria

\begin{tabular}{|l|l|l|l|}
\hline & \multicolumn{3}{|l|}{ Observador } \\
\hline Observador & 2 & 3 & 4 \\
\hline 1 & 0,71 & 0,74 & 0,70 \\
\hline & $(0,56 ; 0,86)$ & $(0,60 ; 0,88)$ & $(0,55 ; 0,86)$ \\
\hline 2 & & 0,84 & 0,74 \\
\hline & & $(0,71 ; 0,97)$ & $(0,59 ; 0,89)$ \\
\hline 3 & & & 0,81 \\
\hline & & & $(0,69 ; 0,94)$ \\
\hline
\end{tabular}


Tabela 5 Valor do índice Kappa ponderado e respectivo intervalo de confiança a 95\% para os quatro observadores em relação à radiografia

\begin{tabular}{|l|l|l|l|}
\hline & Exame & & \\
\hline Observador & Físico & Podoscopia & Baropodometria \\
\hline 1 & 0,23 & 0,18 & 0,19 \\
\hline & $(-0,02 ; 0,48)$ & $(-0,16 ; 0,52)$ & $(-0,11 ; 0,49)$ \\
\hline 2 & 0,15 & 0,00 & 0,00 \\
\hline & $(-0,10 ; 0,40)$ & $(-0,50 ; 0,49)$ & $(-0,37 ; 0,34)$ \\
\hline 3 & 0,15 & 0,13 & 0,00 \\
\hline & $(-0,13 ; 0,43)$ & $(-0,19 ; 0,46)$ & $(-0,32 ; 0,29)$ \\
\hline 4 & 0,25 & 0,15 & 0,13 \\
\hline & $(0,03 ; 0,46)$ & $(-0,14 ; 0,45)$ & $(-0,21 ; 0,48)$ \\
\hline
\end{tabular}

\section{Discussão}

Em virtude do aumento do número de praticantes de corrida, o interesse dos profissionais que realizam o diagnóstico postural vem crescendo. Utilizando métodos como a baropodometria, esses profissionais são capazes de perfazer análises quantitativas com o intuito de compreender melhor os mecanismos que regem a marcha e a corrida, proporcionando, assim, a prevenção de lesões. ${ }^{13}$ A comparação entre os dados fornecidos pelo exame físico, podoscopia, baropodometria com a radiografia com carga, através da mensuração angular já foi estudada pela literatura. ${ }^{14}$ Porém, são escassos os trabalhos que mostram a eficácia da análise de determinados métodos que diagnosticam os diferentes tipos de pé ou pisada. Com esta finalidade, encontramos o relato de Buldt et al., ${ }^{15}$ que classificaram, em seu estudo, os tipos de pé, por meio de medidas antropométricas associadas a uma baropodometria mostrando as diferenças da pressão deste segmento entre os diferentes tipos de pisada.

No presente estudo, avaliamos 40 corredores e numericamente observamos uma amostragem mais baixa de indivíduos com o pé plano, o que corrobora os dados da literatura, ${ }^{15}$ sendo que a maioria dos indivíduos apresenta uma pisada neutra.

Quando analisamos o índice Kappa interobservadores pelo exame físico, notamos uma concordância excelente entre os pesquisadores 1,2 , e 3 . Para tentar interpretar este resultado, foi realizado o desmascaramento dos observadores e inferiu-se que a menor expertise do examinador 4 pudesse ter influenciado a interpretação das variáveis estudadas, apesar de ter sido feita previamente uma explanação prévia a respeito de como deveriam ser realizadas as diferentes análises. Levando-se em consideração a podoscopia, observamos uma boa concordância entre os observadores para o diagnóstico do tipo de pé. Analisando o método da baropodometria isoladamente, notamos que houve uma excelente concordância entre a maioria dos observadores.

De uma forma surpreendente, quando utilizamos as medidas radiográficas para classificar o tipo de pé de acordo com os ângulos de Meary e pitch do calcâneo e comparamos os resultados obtidos através do exame físico, podoscopia, e baropodometria, observamos uma concordância marginal, de acordo com o índice Kappa.

O resultado apresentado mostra-se extremamente interessante nos levando a refletir sobre o diagnóstico do tipo de pé utilizando apenas um método isolado como a baropodometria. Notamos no trabalho de Ward et al. ${ }^{16}$ que a baropodometria como um método isolado de diagnóstico possui baixa reprodutibilidade e aplicabilidade. ${ }^{17}$ Porém, no presente estudo considerando o somatório das informações do exame físico, da podoscopia e da baropodometria, obtivemos uma concordância muito apropriada entre os observadores. Porém, quando comparamos todos os métodos utilizados com os resultados das medidas angulares radiográficas, observamos uma discrepância significativa nos resultados, com isso imaginamos que possa haver uma dificuldade intrínseca em categorizar os diferentes tipos de pés pelas mensurações do pitch do calcâneo e do ângulo de Meary, pois haveria um intervalo muito grande dos valores obtidos para o pé plano e o pé cavo, fazendo com que houvesse uma superestimação dos pés neutros. Talvez se outro método de classificação radiográfica pudesse ser aplicado, um diagnóstico mais preciso do tipo do pé poderia ser alcançado, esperando, com isso, uma melhora nos índices de concordância dos métodos utilizados com a classificação radiográfica.

A segunda possibilidade para discussão se dá ao fato da importância de se realizar uma radiografia com carga para o diagnóstico do tipo do pé através da mensuração angular, servindo, os outros métodos como exame físico, podoscopia e baropodometria como um complemento para o diagnóstico.

Outra vantagem que o uso da radiografia poderia proporcionar seria o de dirimir os erros de uma categorização potencialmente equivocada de classificação podendo ocasionar o uso inadequado de um determinado tipo de calçado ou palmilha causando prejuízo biomecânico e, consequentemente, lesões.

Acreditamos que nosso trabalho apresente alguns aspectos que devem ser aprimorados frente à continuidade deste projeto de pesquisa. Apesar dos resultados obtidos, haveria a necessidade de ampliar a casuística para que um resultado mais robusto pudesse ser obtido. Para tanto, uma amostra ampliada, com base nos resultados de cálculo amostral prévio, e o estudo de diferentes categorias como estudar em separado o sexo envolvido estreitando as faixas etárias poderiam trazer informações mais detalhadas entre os praticantes de corrida, além disso, não avaliamos de forma dinâmica os indivíduos, fato que pode modificar os resultados já que ocorre uma modificação no tipo da pisada nas fases normais da marcha, e estas posições podem ser variáveis entre as pessoas.

\section{Conclusão}

Obtivemos uma concordância excelente entre os observadores ao considerarmos o exame físico, a podoscopia e a baropodometria para o diagnóstico do tipo pisada de corredores. Porém, quando comparados os resultados obtidos do exame físico, baropodometria e podoscopia com as medidas radiográficas, a concordância para o diagnóstico do tipo da pisada foi baixa. 
Conflito de Interesses

Os autores declaram não haver conflito de interesses.

\section{Referências}

1 Neto J Junior, Pastre CM, Monteiro HL. Alterações posturais em atletas brasileiros do sexo masculino que participaram de provas de potência muscular em competições internacionais. Rev Bras Med Esporte 2004;10(03):195-198

2 Lun V, Meeuwisse WH, Stergiou P, Stefanyshyn D. Relation between running injury and static lower limb alignment in recreational runners. Br J Sports Med 2004;38(05):576-580

3 Mutti D. Futsal da iniciação ao alto nível. 2a ed. São Paulo: Phorte; 2003

4 Mattos HM, Pryzsiezny WL. Análise baropodométrica da influência podal na postura. Rev Ter Man Fisioter Manip 2004;3(01): 240-246

5 Guimarães GV, Freitas HF, Silva PR, Teixeira LR. Pés: devemos avaliá-los ao praticar atividade físico-esportiva? Rev Bras Med Esporte 2000;6(02):57-59

6 Nigg BM, Khan A, Fisher V, Stefanyshyn D. Effect of shoe insert construction on foot and leg movement. Med Sci Sports Exerc 1998;30(04):550-555

7 Mansour E, Yaacoub JJ, Bakouny Z, Assi A, Ghanem I. A podoscopic and descriptive study of foot deformities in patients with Down syndrome. Orthop Traumatol Surg Res 2017;103(01):123-127

8 Libotte M. Podoscopíe électronique. Encyclopédie médico-chirurgicale: Kinésithérapie rééducation fonctionnelle. Paris: Elsevier; 2000
9 Stebbins JA, Harrington ME, Giacomozzi C, Thompson N, Zavatsky A, Theologis TN. Assessment of sub-division of plantar pressure measurement in children. Gait Posture 2005;22(04):372-376

10 Leite NM, Faloppa F. Propedêutica ortopédica e traumatológica. Porto Alegre: Atmed; 2013

11 Coughlin MJ, Mann RA, Saltzman CL. Surgery of the foot and ankle. 8th ed. Philadelphia: Mosby-Elsevier; 2007

12 Rosner BA. Fundamentals of biostatistics. 4th ed. Belmont: Duxbury Press; 1995

13 Yang DJ, Park SK, Kim JH, Heo JW, Lee YS, Uhm YH. Effect of changes in postural alignment on foot pressure and walking ability of stroke patients. J Phys Ther Sci 2015;27(09):2943-2945

14 Robinson CC, Balbinot LF, Silva MF, Achaval M, Zaro MA. Plantar pressure distribution patterns of individuals with prediabetes in comparison with healthy individuals and individuals with diabetes. J Diabetes Sci Technol 2013;7(05):1113-1121

15 Buldt AK, Forghany S, Landorf KB, Levinger P, Murley GS, Menz HB. Foot posture is associated with plantar pressure during gait: A comparison of normal, planus and cavus feet. Gait Posture 2018; 62:235-240

16 Ward ED, Phillips RD, Patterson PE, Werkhoven GJ. 1998 William J. Stickel Gold Award. The effects of extrinsic muscle forces on the forefoot-to-rearfoot loading relationship in vitro. Tibia and Achilles tendon. J Am Podiatr Med Assoc 1998;88(10):471-482

17 Baumfeld D, Baumfeld T, da Rocha RL, et al. Reliability of baropodometry on the evaluation of plantar load distribution: a transversal study. BioMed Res Int 2017;2017:5925137 